\title{
Insertional inactivation of the Streptococcus mutans dexA (dextranase) gene results in altered adherence and dextran catabolism
}

\author{
S. M. Colby, ${ }^{1}$ G. C. Whiting, ${ }^{1} \dagger$ L. Tao ${ }^{2} \ddagger$ and R. R. B. Russell ${ }^{1}$ \\ Author for correspondence: S. M. Colby. Tel: +44 191222 7918. Fax: +441912226137. \\ e-mail: S.M.Colby@ newcastle.ac.uk
}

1 Department of Oral Biology, The Dental School, University of Newcastle upon Tyne, Framlington Place, Newcastle upon Tyne NE2 4BW, UK

2 Department of Microbiology and Immunology, University of Oklahoma, Oklahoma City, OK 73190, USA
Streptococcus mutans is able to synthesize extracellular glucans from sucrose which contribute to adherence of these bacteria. Extracellular dextranase can partially degrade the glucans, and may therefore affect virulence of $S$. mutans. In order to isolate mutants unable to produce dextranase, a DNA library was constructed by inserting random Sau3AI-digested fragments of chromosomal DNA from S. mutans into the BamHI site of the streptococcal integration vector pVA891, which is able to replicate in Escherichia coli but does not possess a streptococcal origin of replication. The resultant plasmids were introduced into S. mutans LT11, allowing insertional inactivation through homologous recombination. Two transformants were identified which did not possess dextranase activity. Integration of a single copy of the plasmid into the chromosome of these transformants was confirmed by Southern hybridization analysis. Chromosomal DNA fragments flanking the plasmid were recovered using a marker rescue technique, and sequenced. Comparison with known sequences using the BLASTX program showed $56 \%$ homology at the amino acid level between the sequenced gene fragment and dextranase from Streptococcus sobrinus, strongly suggesting that the $S$. mutans dextranase gene (dexA) had been inactivated. The colony morphology of the dextranase mutants when grown on Todd-Hewitt agar containing sucrose was altered compared to the parent strain, with an apparent build-up of extracellular polymer. The mutants were also more adherent to a smooth surface than LT11 but there was no apparent difference in sucrose-dependent cell-cell aggregation. In contrast to LT11, neither the dexA mutants nor a mutant in the dexB gene, which encodes a dextran glucosidase, were able to ferment dextran to produce acid, supporting an earlier hypothesis that both enzymes are required for metabolism of dextran. From the results obtained by inactivating the dexA gene, a role for dextranase is suggested in controlling the amount and nature of extracellular glucans, in adherence of S. mutans, and in the utilization of glucans as a carbohydrate source.

Keywords: Streptococcus mutans, dextranase, adherence, sucrose metabolism, extracellular enzyme

\section{INTRODUCTION}

The oral streptococci are important in the formation and

†Present address: Department of Medical Microbiology, The Royal Free Hospital, Pond Street, London NW3 2QG, UK.

¥Present address: Department of Oral Biology, University of MissouriKansas City, 650 East 25th Street, Kansas City, MO 64108-2795, USA.

Abbreviation: GTF, glucosyltransferase. metabolic activity of dental plaque, and two species, Streptococcus mutans and Streptococcus sobrinus, are associated with dental caries. Factors which contribute to the virulence of these bacteria include the ability to produce acid from a variety of sugars and the formation of extracellular polymers from sucrose. $S$. mutans produces both water-soluble and water-insoluble glucans from sucrose and these play a significant role in plaque formation as they contribute to the adhesion of this 
species both to the tooth surface and to other bacteria. Water-insoluble glucans, which are particularly important in adherence to hard surfaces, are highly branched polymers consisting predominantly of 1,3 - $\alpha$-linked glucosyl residues with a minor component of $1,6-\alpha-$ linkages (Ebisu et al., 1974). In water-soluble glucans, however, the residues are mainly 1,6- $\alpha$-linked with some 1,3- $\alpha$ - bonds and branch points (Birkhed et al., 1979). The total amount of glucan present, and its structure, is influenced not only by the activity of the three glucosyltransferases (GTF) which produce the glucans (Kuramitsu, 1993), but also by the product of the $\operatorname{dex} A$ gene, an extracellular dextranase which breaks down glucans to isomaltosaccharides by cleaving 1,6- $\alpha$-linkages (Schachtele et al., 1975; Walker et al., 1981; Felgenhauer \& Trautner, 1983). Dextranase may modify glucans by altering the ratio of 1,6- $\alpha$ - to 1,3- $\alpha$-linked chains and hence decrease solubility in water, and may also provide $1,6-\alpha-$ rich fragments to prime further glucan synthesis (Germaine et al., 1977).

In $S$. mutans, dextran is degraded by dextranase to isomaltosaccharides, predominantly isomaltotriose, isomaltotetraose and isomaltopentaose (Dewar \& Walker, 1975; Igarashi et al., 1992). It has been proposed that these oligosaccharides can be transported into the cell and there be degraded to glucose by the product of the $\operatorname{dex} B$ gene, a dextran glucosidase (Russell \& Ferretti, 1990; Whiting et al., 1993). The dexB gene lies in the multiple sugar metabolism ( $m s m)$ operon, which encodes a transport system responsible for the uptake of melibiose, raffinose and isomaltosaccharides and includes $\alpha$-galactosidase, sucrose phosphorylase and the $\operatorname{dex} B$ dextran glucosidase (Russell et al., 1992; Tao et al., 1993b). There has not, however, been any direct demonstration that the extracellular $\operatorname{dex} A$ dextranase and the intracellular $\operatorname{dex} B$ dextran glucosidase function in a coordinated catabolic pathway.

The precise importance of extracellular dextranase in the biology of $S$. mutans is not yet clear but dextranasenegative mutants of $S$. mutans have been shown to be less cariogenic than the parent strain in experimental rats (Tanzer \& Freedman, 1978). It was proposed that the role for dextranase lies in creating a niche for $S$. mutans amongst the complex microflora of dental plaque since $\operatorname{dex} A$ mutants are avirulent in conventional rats but are still fully virulent in mono-infected gnotobiotic rats (Tanzer, 1992). In this report we describe the construction of dextranase mutants of $S$. mutans strain LT11, using the streptococcal integration shuttle vector $\mathrm{pVA} 891$, and experiments undertaken to clarify the contribution of dextranase to sucrose-dependent adherence and the metabolism of glucans.

\section{METHODS}

Bacterial strains. S. mutans strains were grown in Brain Heart Infusion broth (BHI, Oxoid), Todd-Hewitt broth (TH, Oxoid) or the semi-defined medium, CasMM, described by Russell (1979). Erythromycin $\left(15 \mu \mathrm{g} \mathrm{ml} \mathrm{m}^{-1}\right)$ was added when mutants E44, M17 and 522 were cultured to maintain the integrated plasmid pVA891. Eschericbia coli JM109 was grown in LB broth
(Maniatis et al., 1982) with $30 \mu \mathrm{g}$ chloramphenicol $\mathrm{ml}^{-1}$ added when pVA891 was present.

Mutants E44 and M17 were constructed from the transformable S. mutans strain LT11 (Tao et al., 1993a). Mutant 522 was constructed previously by cloning an internal fragment of the $\operatorname{dex} B$ gene into the streptococcal integration vector $\mathrm{pVA} 891$ and transformation of the resulting plasmids into $S$. mutans strain LT11. Recombination between the insert and the homologous gene resulted in insertional inactivation of $\operatorname{dex} B$ (Russell et al., 1992).

Random mutagenesis. $S$. mutans LT11 was mutagenized using the procedure described by Tao et al. (1993c). Chromosomal DNA from $S$. mutans strain GS5 was isolated as described by Ushiro et al. (1991) and completely digested with restriction enzyme Sau3AI. The fragments (mean $0 \cdot 2-0.3 \mathrm{~kb}$ ) were ligated into the BamHI site of the streptococcal integration shuttle vector pVA891 (Macrina et al., 1983). This plasmid expresses resistance to erythromycin in streptococci and $E$. coli, and resistance to chloramphenicol only in $E$. coli, but cannot replicate in streptococci. The resultant pool of recombinant plasmids was used to transform E. coli strain JM109 to chloramphenicol resistance using the calcium chloride method described by Maniatis et al. (1982). The transformants ( $>$ 10000 ) were grown overnight in LB medium containing chloramphenicol. Plasmids were extracted by the method of Birnboim \& Doly (1979) and used to transform S. mutans strain LT11 to erythromycin resistance using the natural transformation method described by Tao et al. (1993a).

Screening for dextranase activity. Approximately 2000 erythromycin-resistant transformants were screened for the ability to hydrolyse high molecular mass dextran by culturing on agar plates containing $0.5 \%$ blue dextran (Donkersloot \& Harr, 1979). Dextranase activity was indicated by a zone of clearing around an area of growth, following incubation for $2 \mathrm{~d}$ in a candle jar at $37^{\circ} \mathrm{C}$. Results were improved if sandwich plates were poured whereby the lower half of the plate contained no blue dextran and the upper half of the plate contained $1 \%$ (w/v) blue dextran.

Southern hybridization. Chromosomal DNA was isolated from dextranase mutants as described above, and 2-3 $\mu \mathrm{g}$ of each DNA was digested to completion with EcoRV, which has a single site in pVA891, and BglII, for which there is no site in the vector. DNA fragments were separated by electrophoresis on a $0.7 \%(\mathrm{w} / \mathrm{v})$ agarose gel. Following electrophoresis, the DNA was denatured as described by Maniatis et al. (1982) and transferred to nylon membranes (Amersham) using a Posiblot transfer apparatus (Stratagene). pVA891 was linearized by digesting with HindIII, and labelled with the Nonradioactive Digoxigenin DNA Labelling and Detection kit (Boehringer Mannheim). This probe was then used in hybridizations under high-stringency conditions according to the manufacturer's instructions.

Marker rescue strategy. DNA flanking the integration vector pVA891 was recovered as described by Tao et al. (1992). Chromosomal DNA from the dextranase mutant M17 was digested with EcoRV, which has a single site in pVA891. The enzyme was heat-inactivated for $20 \mathrm{~min}$ at $65^{\circ} \mathrm{C}$. The DNA mixtures were self-ligated with $\mathrm{T} 4 \mathrm{DNA}$ ligase at $16^{\circ} \mathrm{C}$ then used to transform competent E. coli JM109. Transformants were selected on LB agar containing chloramphenicol $(30 \mu \mathrm{g}$ $\left.\mathrm{ml}^{-1}\right)$. Plasmid DNA was isolated using the method of Birnboim \& Doly (1979) and designated pMEV1. 
Subcloning flanking DNA. The cloned insert in pMEV1 was amplified in a PCR reaction, using oligonucleotide primers based on the sequence of $\mathrm{pVA} 891$ which was originally derived from pACYC184 (Rose, 1988). The primers of sequence $5^{\prime}$ GTC AGG CAC CGT GTA TGA-3' (forward) and 5'-CCC GAT CT'T CCC CAT CG-3' (reverse) were synthesized on an Applied Biosystems synthesizer using the automated phosphoramidite-coupling method. These primers amplified the cloned insert plus approximately $500 \mathrm{bp}$ of vector DNA.

PCR products were purified using a Wizard Clean-up kit (Promega) and then ligated into the PGEM-T vector according to the manufacturer's recommendations (Promega) to produce pTAEV1.

DNA sequencing. Double-stranded template of pTAEV1 was sequenced with pUC/M13 primers, using a Taq cycle sequencing kit (Applied Biosystems). Nucleotide sequence data were manipulated using the PC/Gene software package (IntelliGenetics) and the available databases (March 1994) searched by means of the BLASTX program described by Altschul et al. (1990).

Preparation of dextranase. S. mutans LT11 and mutants M17 and E44 were grown overnight in CasMM supplemented, in the case of the mutants, with $15 \mu \mathrm{g}$ erythromycin $\mathrm{ml}^{-1}$. After centrifugation at 4000 r.p.m. for $15 \mathrm{~min}$ at $4{ }^{\circ} \mathrm{C}$ culture supernatants were concentrated approximately 100 -fold by precipitation with $65 \%(\mathrm{w} / \mathrm{v})$ saturated ammonium sulphate at $4{ }^{\circ} \mathrm{C}$. The precipitate was collected by centrifugation at 10000 r.p.m. for $30 \mathrm{~min}$ at $4{ }^{\circ} \mathrm{C}$, suspended in $0.01 \mathrm{M}$ sodium phosphate buffer (pH 6.0) and dialysed against the same buffer at $4{ }^{\circ} \mathrm{C}$ overnight.

Blue dextran SDSPAGE. Samples were subjected to electrophoresis in SDS-PAGE according to the procedure of Laemmli (1970). Blue dextran $(0.5 \%, \mathrm{w} / \mathrm{v})$ was incorporated into the $8 \%$ $(\mathrm{w} / \mathrm{v})$ acrylamide resolving gel mix before addition of crosslinking and catalyst agents (Barrett \& Curtiss, 1986). Samples were loaded and electrophoresed at $100 \mathrm{~V}$ for $1-2 \mathrm{~h}$ at room temperature. Dextranase activity was detected as cleared zones after the gel was washed in several changes of $50 \mathrm{mM}$ Tris $/ \mathrm{HCl}$, $\mathrm{pH} 7.5$, then incubated overnight at $37^{\circ} \mathrm{C}$ in $0.1 \mathrm{M}$ sodium phosphate buffer, $\mathrm{pH} 6.0$, containing $1 \%(\mathrm{v} / \mathrm{v})$ Triton X-100 to remove residual SDS.

Adherence assay. A modification of the method described by Larrimore et al. (1983) was used. S. mutans strains were grown overnight in CasMM with antibiotic added where appropriate then diluted 1:30 in CasMM containing 0.5\% (w/v) sucrose in a 24-well tissue culture tray. Exogenous dextranase from Penicillium sp. (0.25 U; Sigma) was added at this stage if required. After anaerobic incubation for $24 \mathrm{~h}$ at $37^{\circ} \mathrm{C}$, growth medium was gently removed and the tray washed twice with water to remove nonadherent cells. Attached cells were stained with a $0.5 \%$ crystal violet solution for $5 \mathrm{~min}$, washed several times with water and photographed before drying.

Bacterial aggregation assay. Cell-cell aggregation was measured in CasMM in the presence and absence of $0.5 \%(\mathrm{w} / \mathrm{v})$ sucrose, as described by Douglas \& Russell (1982).

Dextran fermentation. $S$. mutans LT11, the $\operatorname{dex} A$ mutants E44 and $\mathrm{M} 17$, and the dexB mutant 522 , were tested for the ability to ferment dextran T10 and dextran T2000 (both obtained from Pharmacia). Cells from overnight $\mathrm{TH}$ cultures were washed twice in sterile saline and adjusted to an $\mathrm{OD}_{620}$ of $1.0 ; 25 \mu \mathrm{l}$ was used to inoculate basal medium containing purple base broth (16 $\mathrm{g} \mathrm{l}^{-1}$, Difco) and thioglycollate medium (24 $\mathrm{g} \mathrm{l}^{-1}$, Difco) (Shklair \& Keene, 1974) supplemented with $0.5 \%$ (w/v) dextran, in a microtitre tray. After incubation at $37^{\circ} \mathrm{C}$ for $24 \mathrm{~h}$ in a candle jar, a positive fermentation result was indicated by a colour change from purple to yellow.
To quantify production of acid, cells were grown overnight in $\mathrm{TH}$ broth then inoculated to an $\mathrm{OD}_{620}$ of 0.2 into fresh $\mathrm{TH}$ broth supplemented with $0.5 \%(\mathrm{w} / \mathrm{v}$ ) dextran T10 (Pharmacia). After overnight incubation at $37^{\circ} \mathrm{C}$ the $\mathrm{pH}$ of the culture supernatant was measured.

\section{RESULTS}

\section{Production of dexA mutants}

The mutagenesis procedure results in the construction of a collection of erythromycin-resistant transformants of $S$. mutans LT11 in which pVA891 has been inserted at random sites on the chromosome, the site of insertion in each being determined by the cloned Sau3AI fragment. No erythromycin-resistant colonies were obtained when pVA891 alone was introduced into LT11 showing that the insertion of the vector into the chromosome was not a random event.

Approximately 2000 erythromycin-resistant transformants were screened on blue dextran agar plates for the ability to produce dextranase and two transformants which showed no dextranase activity, E44 and M17, were detected. Southern hybridization analysis, using pVA891 as a probe, revealed that inactivation resulted from integration of a single copy of the vector into the chromosome of each mutant. This indicated that a single gene had been inactivated but it was necessary to determine whether this was the $\operatorname{dex} A$ structural gene or a regulatory gene which could result in the same phenotype.

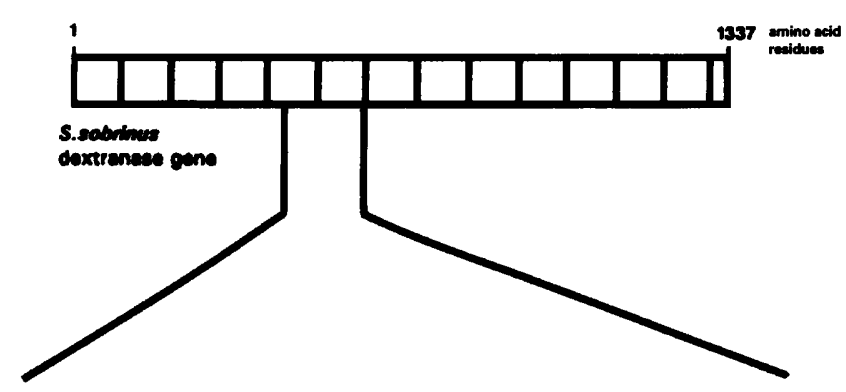

QRYYNPTSXIWODY IADRYOAMKNGGFDGWOGDTIGDNEVYSYADXOSNDPS : : : : : : : : : : : : : : : : : : : : : : : : : : : : : : : : : : : : :

KKFWLTEGYAEFLRAI KEKC PNYYLTVNDVNGEOI YRL KDGNODVI YNEIWPF

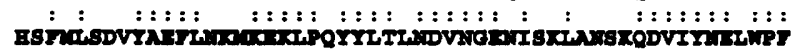

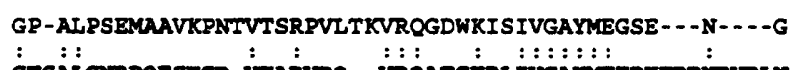

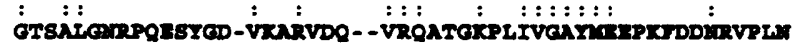

GSKADAEAGKSLQTDAVLLTSASIAAA

: : : : : ::::: : : : : :

Fig. 1. Amino acid homology between the recovered gene fragment from $S$. mutans (lower sequence) and an internal fragment of $S$. sobrinus dextranase sequenced by Wanda \& Curtiss (1994) (upper sequence). 

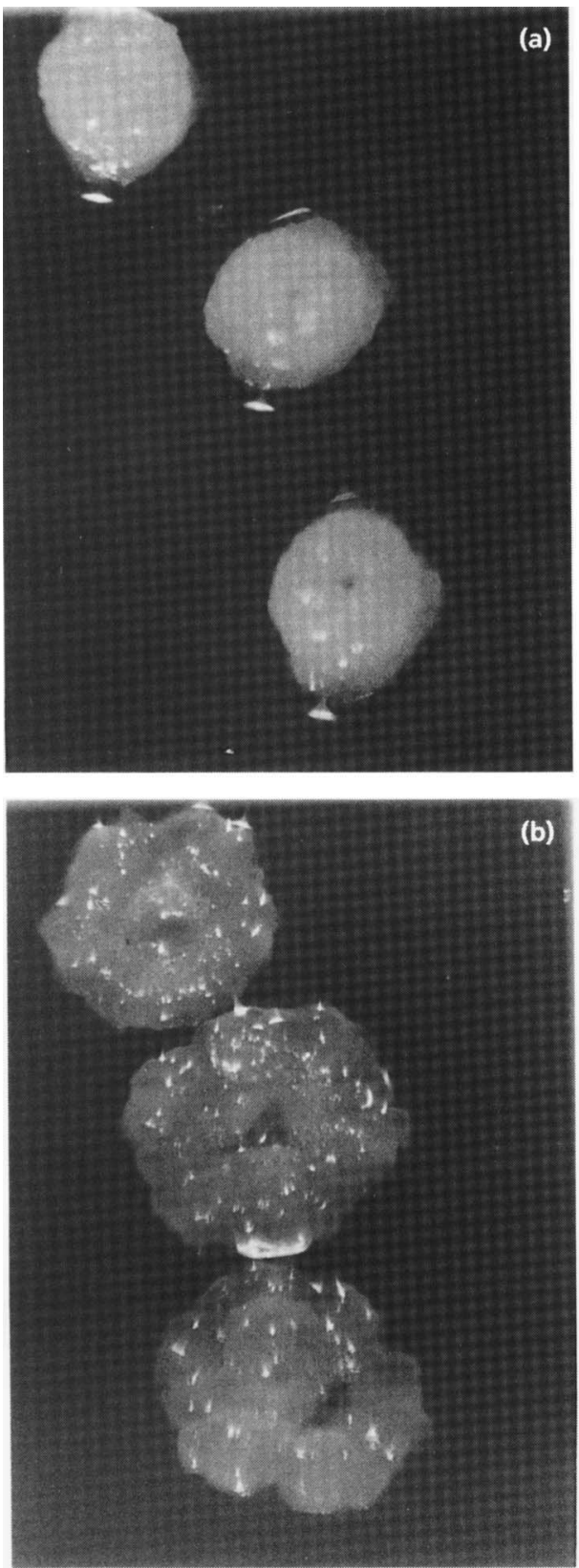

Fig. 2. Colony morphology of S. mutans LT11 (a) and the dexA mutant M17 (b) on TH agar supplemented with sucrose. Plates were incubated anaerobically at $37^{\circ} \mathrm{C}$ for $48 \mathrm{~h}$ then aerobically at $4^{\circ} \mathrm{C}$ for $3 \mathrm{~d}$.

Stability studies were not carried out on the mutants but all subculturing was performed in the presence of erythromycin to ensure the phenotype was maintained.

Using a marker rescue strategy, DNA flanking one side of the integration vector $\mathrm{pVA} 891$ was recovered by digesting chromosomal DNA from mutant M17 with EcoRV, which has a single site in the vector. This resulted in a fragment consisting of most of the vector plus flanking DNA, which was recircularized to produce pMEV1. The insert DNA was then amplified by PCR using primers

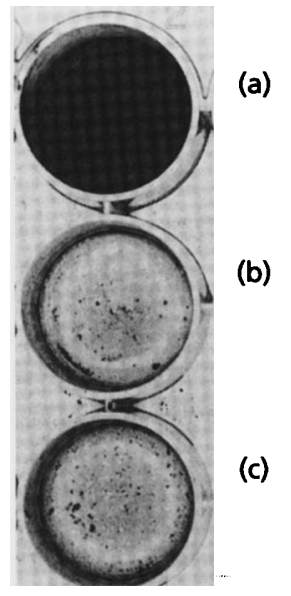

Fig. 3. Accumulation of S. mutans LT11 and the $\operatorname{dexA}$ mutant M17 on a plastic tissue culture tray: (a) M17, (b) M17 plus exogenous dextranase from Penicillium sp. and (c) LT11. After growth, unattached cells were removed by washing and adherent cells were stained with $0.5 \%$ crystal violet.

based on the vector sequence. The PCR product was cloned into pGEM-T to produce pTAEV1, and from this it was possible to sequence $534 \mathrm{bp}$ of the inactivated gene. The BLASTX program was used to search available databases and greatest amino acid homology $(56 \%)$ was found between this sequence and the $\operatorname{dex} A$ dextranase gene from S. sobrinus (Fig. 1). This was the only sequence in the databases searched which showed significant homology with our sequence. A similar result was obtained for E44, strongly suggesting that the $\operatorname{dex} A$ gene had been inactivated in both mutants.

\section{Characterization of dextranase mutants}

When crude dextranase preparations were analysed by blue dextran SDS-PAGE, three bands of activity were seen in the $S$. mutans LT11 sample with apparent molecular masses of $167 \mathrm{kDa}, 108 \mathrm{kDa}$ and $96 \mathrm{kDa}$. No active bands appeared in the lanes containing the mutant samples E44 and M17.

The colony morphology of both E44 and M17 differed from that of $S$. mutans LT11 when grown on TH plates supplemented with $1 \%(\mathrm{w} / \mathrm{v})$ sucrose. Colonies were allowed to grow for $48 \mathrm{~h}$ at $37^{\circ} \mathrm{C}$ and plates were then transferred to $4{ }^{\circ} \mathrm{C}$. Colonies of the dextranase mutants were surrounded by large translucent droplets which were not seen around the wild-type. After $3 \mathrm{~d}$ at $4{ }^{\circ} \mathrm{C}$ there was a marked difference in size between $S$. mutans LT11 and the dextranase mutants with a build-up of extracellular polymer around the latter (Fig. 2). There was no difference between $S$. mutans LT11 and the dextranase mutants grown on TH agar which had not been supplemented with sucrose.

Mutants E44 and M17 showed markedly greater accumulation on the surface of plastic plates than $S$. mutans LT11 when grown in the presence of sucrose (Fig. 3). 


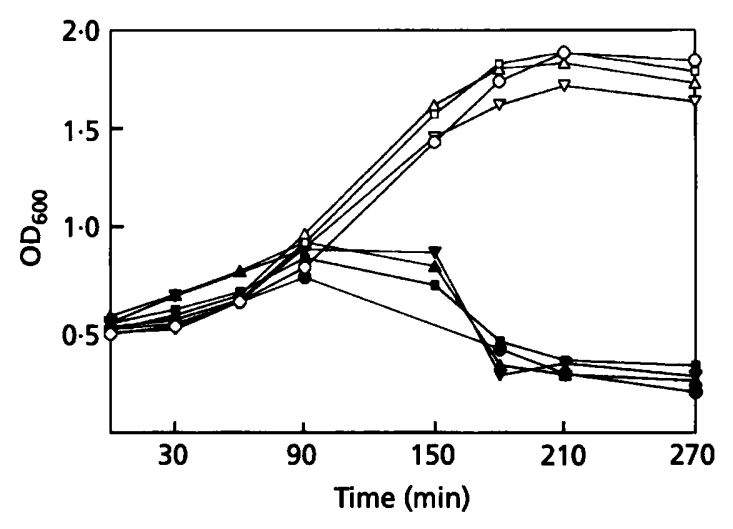

Fig. 4. Bacterial aggregation in the presence and absence of sucrose measured by the $O_{600}$ change of cell suspensions. Sucrose was added at time 0. Strains used: LT11 $(0,0)$; E44 ( $\square$, $\square) ;$ M17 $(\Delta, \triangle) ; 522(\nabla, \nabla)$. Filled symbols, sucrose added; open symbols, no sucrose added.

However, when exogenous dextranase from Penicillium sp. was added to the mutant cultures, the level of adherence of the three strains was comparable (Fig. 3). A similar effect was achieved if the cell-free supernatant of an $S$. mutans LT11 culture, also grown in CasMM, was added to the mutant cultures. Since the LT11 supernatant contains extracellular dextranase the indication is that the increased accumulation of the mutants was due to the effect that inactivating the $\operatorname{dex} A$ gene had on the nature and amount of glucan produced. No difference was found between $S$. mutans LT11 and the dextranase mutants when they were compared for the ability to aggregate in the presence of sucrose (Fig. 4).

\section{DexA/DexB pathway of dextran catabolism}

It has been proposed that dextran can be degraded to glucose by $S$. mutans via a pathway involving both the $\operatorname{dex} A$ and $\operatorname{dex} B$ gene products (Russell \& Ferretti, 1990; Whiting et al., 1993). This was supported by the fact that the dextranase mutants E44 and M17 were unable to produce acid from fermentation of dextran T10 whereas the effect of adding dextran T10 to a culture of $S$. mutans LT11 was to give a fall in pH of $0 \cdot 26 \pm 0 \cdot 04(n=3)$. LT11 was also able to ferment dextran T2000 although this was a slow process requiring several days incubation. Neither the $\operatorname{dex} A$ mutants E44 and M17, nor a dexB mutant, strain 522 , described by Whiting et al. (1993), were able to produce acid from fermentation of dextran T2000.

\section{DISCUSSION}

The results presented in this paper show that $S$. mutans LT11 produces active dextranase in multiple forms of $167 \mathrm{kDa}, 108 \mathrm{kDa}$ and $96 \mathrm{kDa}$ when analysed by blue dextran SDS-PAGE. Mutants E44 and M17, created using the streptococcal integration vector $\mathrm{pVA891, \text {were }}$ unable to hydrolyse dextran, and Southern hybridization using pVA891 as a probe showed that a single copy of the vector had integrated into the $S$. mutans chromosome. Since this integration event resulted in complete loss of dextranase activity the indication is that a single copy of $\operatorname{dex} A$ is present in $S$. mutans as has been found to be the case in S. sobrinus (Barrett et al., 1986) and Streptococcus salivarius (Lawman \& Bleiweis, 1991). Multiple forms of dextranase have previously been reported for S. sobrinus (Barrett et al., 1987), S. mutans (Russell \& Ferretti, 1990; Igarashi et al., 1992) and S. salivarius (Lawman \& Bleiweis, 1991). The higher molecular mass forms of $S$. mutans dextranase activity gradually broke down during storage to smaller forms still retaining activity (Russell \& Ferretti, 1990) although Igarashi et al. (1992) successfully inhibited breakdown of the larger product with a protease inhibitor. The variation in band sizes may thus be explained by proteolytic degradation and Barrett et al. (1986) observed that whereas high molecular mass forms of $S$. sobrinus dextranase were produced in all growth phases, low molecular mass forms varied during culture growth phase coinciding with protease activity. Proteases have recently been demonstrated in $S$. mutans (Harrington \& Russell, 1994) but whether the cleavage of dextranase occurs at specific sites, and whether the processing has functional significance, is not yet known. The true size of dextranase can only be determined from the deduced primary sequence, particularly since intact dextranase shows anomalous migration on SDS-PAGE; Wanda \& Curtiss (1994) calculated the molecular mass of $S$. sobrinus dextranase to be $140 \mathrm{kDa}$ whilst active bands with apparent molecular mass as high as $175 \mathrm{kDa}$ were visualized on SDS-PAGE gels. Igarashi et al. (1995) have determined the nucleotide sequence of $S$. mutans $\operatorname{dex} A$ and deduced the molecular mass to be $94.5 \mathrm{kDa}$ so it appears that $S$. mutans dextranase also shows anomalous migration.

Nucleotide sequencing of part of the inactivated gene flanking the inserted plasmid revealed that it encoded a stretch of protein with $56 \%$ homology with an internal fragment of the $S$. sobrinus dextranase recently reported by Wanda \& Curtiss (1994). The entire S. mutans dextranase gene sequence has now been determined (Igarashi $e t$ al., 1995) and submitted to GenBank (accession number D49430) and there is $>45 \%$ overall amino acid homology between this gene and the dextranase gene from S. sobrinus. The sequence reported by us shows complete homology with the corresponding middle region of the gene sequenced by Igarashi et al. (1995).

Despite the similarity in sequence, however, it appears that dextranase in $S$. mutans is regulated in an entirely different way to $S$. sobrinus, in which a dextranase inhibitor plays a role in controlling activity (Hamelik \& McCabe, 1982; Sun et al., 1994; Wellington et al., 1994). No comparable inhibitor has been reported in $S$. mutans and our investigation has failed to reveal one.

The colony morphology of the $\operatorname{dex} A$ mutants on agar containing sucrose was altered compared to the wild-type, with more extracellular polymer around the mutant colonies. Altered colony morphology can result from mutations in the genes responsible for the synthesis of 
glucans, or the other extracellular polymer produced from sucrose, fructan (de Stoppelaar et al., 1971; Okahashi et al., 1984). In this study, inactivation of the $\operatorname{dex} A$ gene resulted in altered colony morphology and this is also believed to be due to changes in the nature and structure of the extracellular glucans, in this case due to an increase in 1,6- $\boldsymbol{\alpha}$-linked glucosyl residues. Tanzer \& Freedman (1978) produced dextranase mutants by chemical mutagenesis but found no differences in colonial morphology on sucrose-containing agar between wild-type and their mutants. This observation may have been a consequence of the media chosen. In the present study, whilst differences between the mutants and parent strain were noted on all sucrose-containing agars tested, the effect was most pronounced on sucrose-supplemented TH agar. Furthermore, the difference between the wild-type and mutants became more apparent after extended incubation times.

Since dextranase plays a role in modification of extracellular polymers, absence of this enzyme would be expected to affect the attachment of the bacteria. Adherence assays indicated that this was the case as the mutants accumulated on the surface of tissue-culture wells to a markedly greater extent than did the parent strain. That this increased accumulation resulted from loss of dextranase was confirmed by addition of exogenous commercial dextranase or cell-free culture supernatant from $S$. mutans LT11, both of which restored the wildtype phenotype. Ebisu et al. (1974) compared the properties of water-insoluble glucans made in the presence and absence of exogenous dextranase. The glucan formed in the presence of dextranase differed from the native glucan in that it was essentially linear with few 1,6- $\alpha$-linkages, and although water-insoluble it was nonadherent. Their findings indicated that 1,6- $\alpha$-linkages and branching are responsible for the adhesive nature of the glucan whereas water-insolubility is a consequence of the 1,3- $\alpha$-linked backbone. In the present study, the mutants lacked endogenous dextranase so the glucan produced will have more 1,6- $\alpha$-linkages than the wild-type glucan. The increased 1,6- $\alpha$-glucan would account for the apparent greater bulk of the mutant colonies and the increased cell mass accumulating on hard surfaces.

Although adherence to a surface was altered in the mutants, there was no effect on sucrose-induced aggregation in CasMM (Fig. 4) or TH broth (data not shown). It has, however, been reported that exogenous dextranase decreases cell-cell aggregation (Graves \& Verran, 1984) so the extent of aggregation may be susceptible to modulation by very high levels of dextranase activity. Also, it is not known what effect inactivating the $\operatorname{dex} A$ gene would have on glucan-mediated aggregation of $S$. mutans with other bacterial species, which would occur in vivo. Dextranase activity is thus one of the factors capable of influencing the sucrose-dependent cellsurface and cell-cell adherence properties of $S$. mutans. The properties of the glucan produced from sucrose under any particular set of conditions are determined by the relative levels of activity of each of the three GTFs, each of which produces a glucan with distinctive properties (Walker et al., 1990). Since one GTF may modify glucans made by other GTFs, there is the potential for creation of an enormous variety of products displaying a range of functional differences determined by the varying arrangement of 1,6- $\alpha$ - and 1,3- $\alpha$,-linkages. Dextranase further modifies nascent and completed glucans to influence the final structure (Germaine et al., 1977). The relative importance of each GTF and dextranase appears to vary in different strains of $S$. mutans since it is known that GTF activity for both water-soluble and waterinsoluble glucans is highly strain dependent (Montville et al., 1977). In dental plaque, an even more complex sequence of events will therefore be taking place, since most individuals carry several distinct clonotypes of $S$. mutans, as well as other species which also produce glucans and dextranases.

Chemically induced mutants lacking dextranase have been reported to be less cariogenic in rats than the wild-type $S$. mutans, a finding which might seem at variance with our observation that dextranase mutants are more adherent than the parent strain. However, adherence is only one of the properties determining the ability of $S$. mutans to colonize and Tanzer (1992) has proposed that an important function of dextranase is to degrade the glucan in plaque and enhance the ability of $S$. mutans to establish itself. This would explain the failure of $\operatorname{dex} A$ mutants to cause caries in rats with a normal oral flora, yet still be fully virulent when used to monoinfect. Lack of dextranase in the mutants would make them less competitive with other plaque bacteria, though whether this is due to the inability to invade established plaque or to altered adherence properties is not clear.

The possible function of dextranase in the utilization of extracellular glucans as a storage polysaccharide has long been recognized (Parker \& Creamer, 1971) but the detailed mechanism has received little attention. Shortchain isomaltosaccharides produced by the action of dextranase on glucans can be transported into the cell and further degraded to glucose by DexB glucosidase (Russell \& Ferretti, 1990; Whiting et al., 1993). S. mutans LT11 produces acid as a result of dextran fermentation but neither $\operatorname{dex} A$ mutants (this study) nor $\operatorname{dex} B$ mutants (Whiting et al., 1993) are able to do this. These observations indicate that a pathway involving extracellular dextranase and intracellular dextran glucosidase exists, enabling glucans to serve as a carbon source. This may confer an ecological advantage on $S$. mutans as other oral streptococci are unable to ferment dextrans (Lawman \& Bleiweis, 1991; our unpublished observations).

In conclusion, gene inactivation studies have indicated that extracellular dextranase is important not only in modulating the adhesive properties of glucans but also in degradation of dextran for use as a fermentable carbohydrate source. Both of these aspects are likely to be of importance in allowing $S$. mutans to colonize the tooth surface and compete effectively with other dental plaque bacteria. 


\section{ACKNOWLEDGEMENTS}

This work was supported by Medical Research Council grant G9007878CB and Public Service grant DE08191 from the US National Institutes of Health.

\section{REFERENCES}

Altschul, S. F., Gish, W., Miller, W., Myers, E. W. \& Lipman, D. J. (1990). Basic local alignment search tool. J Mol Biol 215, 403-410.

Barrett, J. F. \& Curtiss, R., III (1986). Renaturation of dextranase activity from culture supernatant fluids of Streptococcus sobrinus after sodium dodecylsulfate polyacrylamide gel electrophoresis. Anal Biochem 158, 365-379.

Barrett, J. F., Barrett, T. A. \& Curtiss, R., III (1986). Biochemistry and genetics of dextranase from Streptococcus mutans 6715. In Molecular Microbiology and Immunology of Streptococcus mutans, pp. 205-215. Edited by S. Hamada, S. M. Michalek, H. Kiyono, L. Menaler \& J. R. McGhee. Amsterdam: Elsevier Science Publishers.

Barrett, J. F., Barrett, T. A. \& Curtiss, R., III (1987). Purification and partial characterization of the multicomponent dextranase complex of Streptococcus sobrinus and cloning of the dextranase gene. Infect Immun 55, 792-802.

Birkhed, D., Rosell, K.-G. \& Granath, K. (1979). Structure of extracellular water-soluble polysaccharides synthesized from sucrose by oral strains of Streptococcus mutans, Streptococcus salivarius, Streptococcus sanguis and Actinomyces viscosus. Arch Oral Biol 24, 53-61.

Birnboim, H. C. \& Doly, J. (1979). A rapid alkaline extraction procedure for screening recombinant plasmid DNA. Nucleic Acids Res 7, 1513-1523.

Dewar, M. D. \& Walker, G. J. (1975). Metabolism of the polysaccharides of human dental plaque. I. Dextranase activity of streptococci, and the extracellular polysaccharides synthesized from sucrose. Caries Res 9, 21-35.

Donkersloot, J. A. \& Harr, R. J. (1979). More sensitive test agar for detection of dextranase-producing oral streptococci and identification of two glucan synthesis-defective dextranase mutants of Streptococcus mutans 6715. J Clin Microbiol 10, 919-922.

Douglas, C. W. I. \& Russell, R. R. B. (1982). Effect of specific antisera on adherence properties of the oral bacterium Streptococcus mutans. Arch Oral Biol 27, 1039-1045.

Ebisu, S., Misaki, A., Kato, K. \& Kotani, S. (1974). The structure of water-insoluble glucans of cariogenic Streptococcus mutans, formed in the absence and presence of dextranase. Carbobydr Res 38, 374-381.

Felgenhauer, B. \& Trautner, K. (1983). The reciprocal action of extracellular $S$. mutans glucosyltransferase and glucanhydrolase. $J$ Dent Res 62, 459.

Germaine, G. R., Harlander, S. K., Leung, W.-L. S. \& Schachtele, C. F. (1977). Streptococcus mutans dextransucrase: functioning of primer dextran and endogenous dextranase in water-soluble and waterinsoluble glucan synthesis. Infect Immun 16, 637-648.

Graves, W. \& Verran, J. (1984). Effect of dextranase and protease enzymes on aggregation of Streptococcus mutans: colorimetric and electron microscopic studies. Microbios 40, 145-152.

Hamelik, R. M. \& McCabe, M. M. (1982). An endodextranase inhibitor from batch cultures of Streptococcus mutans. Biochem Biopbys Res Commun 106, 875-880.

Harrington, D. J. \& Russell, R. R. B. (1994). Identification and characterisation of two extracellular proteases of Streptococcus mutans. FEMS Microbiol Lett 121, 237-242.

Igarashi, T., Yamamoto, A. \& Goto, N. (1992). Characterization of the dextranase purified from Streptococcus mutans Ingbritt. Microbiol Immunol 36, 969-976.

Igarashi, T., Yamamoto, A. \& Goto, N. (1995). Sequence analysis of the Streptococcus mutans Ingbritt $\operatorname{dex} A$ gene encoding extracellular dextranase. Microbiol Immunol (in press).

Kuramitsu, H. K. (1993). Virulence factors of mutans streptococci: role of molecular genetics. Crit Rev Oral Biol Med 4, 159-176.

Laemmli, U. K. (1970). Cleavage of structural proteins during the assembly of the head of bacteriophage T4. Nature 227, 680-685.

Larrimore, S., Murchison, H., Shiota, T., Michalek, S. M. \& Curtiss, R., III (1983). In vitro and in vivo complementation of Streptococcus mutans mutants defective in adherence. Infect Immun 42, 558-566.

Lawman, P. \& Bleiweis, A. S. (1991). Molecular cloning of the extracellular endodextranase of Streptococcus salivarius. J Bacteriol 173, 7423-7428.

Macrina, F. L., Evans, R. P., Tobian, J. A., Hartley, D. L., Clewell, D. B. \& Jones, K. R. (1983). Novel shuttle plasmid vehicles for Eschericbia-Streptococcus transgeneric cloning. Gene 25, 145-150.

Maniatis, T., Fritsch, E. F. \& Sambrook, J. (1982). Molecular Cloning: a Laboratory Manual. Cold Spring Harbor, NY : Cold Spring Harbor Laboratory.

Montville, T. J., Cooney, C. L. \& Sinskey, A. J. (1977). Measurement and synthesis of insoluble and soluble dextran by Streptococcus mutans. J Dent Res 56, 983-989.

Okahashi, N., Asakawa, H., Koga, T., Masuda, N. \& Hamada, S. (1984). Clinical isolates of Streptococcus mutans serotype $c$ with altered colony morphology due to fructan synthesis. Infect Immun 44, 617-622.

Parker, R. B. \& Creamer, H. R. (1971). Contribution of plaque polysaccharides to growth of cariogenic microorganisms. Arch Oral Biol 16, 855-862.

Rose, R. E. (1988). The nucleotide sequence of pACYC184. Nucleic Acids Res 16, 355.

Russell, R. R. B. (1979). Purification of Streptococcus mutans glucosyltransferase by polyethylene glycol precipitation. FEMS Microbiol Lett 6, 197-199.

Russell, R. R. B. \& Ferretti, J. J. (1990). Nucleotide sequence of the dextran glucosidase $(\operatorname{dex} B)$ gene of Streptococcus mutans. J Gen Microbiol 136, 803-810.

Russell, R. R. B., Aduse-Opoku, J., Sutcliffe, I. C., Tao, L. \& Ferretti, J. J. (1992). A binding protein-dependent transport system in Streptococcus mutans responsible for multiple sugar metabolism. J Biol Chem 267, 4631-4637.

Schachtele, C. F., Staat, R. H. \& Harlander, S. K. (1975). Dextranases from oral bacteria: inhibition of water-insoluble glucan production and adherence to smooth surfaces by Streptococcus mutans. Infect Immun 12, 309-317.

Shklair, l. L. \& Keene, H. J. (1974). A biochemical scheme for the separation of the five varieties of Streptococcus mutans. Arcb Oral Biol 19, 1079-1081.

de Stoppelaar, J. D., Konig, K. G., Plasschaert, A. J. M. \& van der Hoeven, J. S. (1971). Decreased cariogenicity of a mutant of Streptococcus mutans. Arch Oral Biol 16, 971-975.

Sun, J.-W., Wanda, S.-Y., Camilli, A. \& Curtiss, R., III (1994). Cloning and DNA sequencing of the dextranase inhibitor gene (dei) from Streptococcus sobrinus. J Bacteriol 176, 7213-7222.

Tanzer, J. M. (1992). Microbiology of dental caries. In Contemporary Oral Microbiology and Immunology, pp. 377-424. Edited by J. Slots \& M. A. Taubman. St Louis, MO: Mosby-Year Book.

Tanzer, J. M. \& Freedman, M. L. (1978). Genetic alterations of Streptococcus mutans' virulence. Adv Exp Med Biol 107, 661-672. 
Tao, L., LeBlanc, D. J. \& Ferretti, J. J. (1992). Novel streptococcalintegration shuttle vectors for gene cloning. Gene 120, 105-110.

Tao, L., MacAlister, T. J. \& Tanzer, J. M. (1993a). Transformation efficiency of EMS-induced mutants of Streptococcus mutans of altered cell shape. J Dent Res 72, 1032-1039.

Tao, L., Sutcliffe, I. C., Russell, R. R. B. \& Ferretti, J. J. (1993b). Transport of sugars, including sucrose, by the $m s m$ transport system of Streptococcus mutans. J Dent Res 72, 1386-1390.

Tao, L., Tanzer, J. M., Kuramitsu, H. K. \& Das, A. (1993c). Identification of several rod loci and cloning of the rodD locus of Streptococcus mutans. Gene 126, 123-128.

Ushiro, I., Lumb, S. M., Aduse-Opoku, J., Ferretti, J. J. \& Russell, R. R. B. (1991). Chromosomal deletions in melibiose-negative isolates of Streptococcus mutans. J Dent Res 70, 1422-1426.

Walker, G. J., Pulkownik, A. \& Morrey-Jones, J. G. (1981). Metabolism of the polysaccharides of human dental plaque: release of dextranase in batch cultures of Streptococcus mutans. $J \mathrm{Gen}$ Microbiol 127, 201-208.
Walker, G. J., Cheetham, N. W. H., Taylor, C., Pearce, B. J. \& Slodki, M. E. (1990). Productivity of four $\alpha$-D-glucosyltransferases released by Streptococcus sobrinus under defined conditions in continuous culture. Carbobydr Polym 13, 399-421.

Wanda, S.-Y. \& Curtiss, R., III (1994). Purification and characterization of Streptococcus sobrinus dextranase produced in recombinant Escherichia coli and sequence analysis of the dextranase gene. $J$ Bacteriol 176, 3839-3850.

Wellington, J. E., Shaw, J. M. \& Walker, G. J. (1994). Influence of growth rate on the relative activities of free and bound dextranase and dextranase inhibitor in continuous cultures of Streptococcus sobrinus. Microbios 121, 121-129.

Whiting, G. C., Sutcliffe, I. C. \& Russell, R. R. B. (1993). Metabolism of polysaccharides by the Streptococcus mutans $\operatorname{dexB}$ gene product. $J$ Gen Microbiol 139, 2019-2026.

Received 18 April 1995; revised 27 June 1995; accepted 11 July 1995. 